\title{
Which Patients with Isolated Para-aortic Lymph Node Metastasis Will Truly Benefit from Extended Lymph Node Dissection for Colon Cancer?
}

\author{
Sung Uk Bae, MD, MS 1,2 \\ Hyuk Hur, MD, PhD² \\ Byung Soh Min, MD, PhD² \\ Seung Hyuk Baik, MD, PhD² \\ Kang Young Lee, MD, PhD² \\ Nam Kyu Kim, MD, PhD, FACS²
}

${ }^{1}$ Division of Colorectal Surgery, Department of Surgery, Dongsan Medical Center, Keimyung University School of

Medicine, Daegu, ${ }^{2}$ Division of

Colorectal Surgery, Department of Surgery, Colorectal Cancer Clinic, Severance Hospital, Yonsei University College of Medicine, Seoul, Korea

Correspondence: Nam Kyu Kim, MD, PhD, FACS Division of Colorectal Surgery,

Department of Surgery, Colorectal Cancer Clinic,

Severance Hospital, Yonsei University College

of Medicine, 50-1 Yonsei-ro, Seodaemun-gu,

Seoul 03722, Korea

Tel: 82-2-2228-2117

Fax: 82-2-313-8289

E-mail: namkyuk@yuhs.ac

Received February 24, 2017

Accepted July 6, 2017

Published Online July 14, 2017

*This study was presented at the 42th Annual Meeting of the Korean Cancer Association, June 16-17, 2016, Seoul, Korea.

\begin{abstract}
Purpose
The prognosis of patients with colon cancer and para-aortic lymph node metastasis (PALNM) is poor. We analyzed the prognostic factors of extramesenteric lymphadenectomy for colon cancer patients with isolated PALNM.
\end{abstract}

\section{Materials and Methods}

We retrospectively reviewed 49 patients with PALNM who underwent curative resection between October 1988 and December 2009.

\section{Results}

In univariate analyses, the 5-year overall survival (OS) and disease-free survival (DFS) rates were higher in patients with $\leq 7$ positive para-aortic lymph node (PALN) $(36.5 \%$ and $27.5 \%)$ than in those with > 7 PALN (14.3\% and 14.3\%; $p=0.010$ and $p=0.027$, respectively), and preoperative carcinoembryonic antigen (CEA) level $>5$ was also correlated with a lower 5-year OS and DFS rate of $21.5 \%$ and $11.7 \%$ compared with those with CEA $\leq 5$ (46.3\% and $41.4 \% ; p=0.122$ and 0.039 , respectively). Multivariate analysis found that the number of positive PALN (hazard ratio [HR], 3.291; 95\% confidence interval [Cl], 1.309 to 8.275; $\mathrm{p}=0.011$ ) was an independent prognostic factor for OS and the number of positive PALN (HR, 2.484; 95\% Cl, 0.993 to 6.211; $p=0.052$ ) and preoperative CEA level (HR, 1.953; 95\% $\mathrm{Cl}, 0.940$ to 4.057 ; $\mathrm{p}=0.073$ ) were marginally independent prognostic factors for DFS. According to our prognostic model, the 5-year OS and DFS rate increased to $59.3 \%$ and $53.3 \%$, respectively, in patients with $\leq 7$ positive PALN and CEA level $\leq 5$.

\section{Conclusion}

PALN dissection might be beneficial in carefully selected patients with a low CEA level and less extensive PALNM.

\section{Introduction}

Isolated para-aortic lymph node (PALN) metastasis (PALNM) occurs in up to $2 \%$ of colorectal cancer (CRC) patients and is regarded as a poor prognostic factor $[1,2]$. Although the American Joint Committee on Cancer (AJCC) staging system classifies CRC with metastasis to PALNs as M1 disease, the clinical characteristics of it have not been comprehensively elucidated and there is no consensus on the
Key words

Colonic neoplasms, Lymph nodes, Lymph node excision, Lymphatic metastasis treatment strategy for isolated PALNM in CRC [3].

The complete response rate in colon cancer patients with PALNM remains low despite newly developed chemotherapeutic agents and it is difficult to control the progression of disease without surgical intervention [1]. In CRC patients with hepatic or pulmonary metastases, aggressive hepatic or lung resection has become a standard strategy when R0 resection can be achieved. However, the role of PALN dissection (PALND) has not been established due to its low incidence, postoperative morbidity, and advances in the field 
of chemotherapy.

Previously, we reported that the oncologic outcomes of colon cancer with isolated PALNM were comparable with outcomes of metastatectomy for liver metastasis and suggested that upfront primary tumor resection with extended lymphadenectomy may be justified in selected patients [4]. However, only a few patients have any benefit from extensive dissection and the key factors in subsets of patients with PALNM that may experience oncologic benefits from PALND remain unknown.

The description of prognostic factors for pathological PALNM allows the identification of patients who may benefit from PALND while sparing other patients from the morbidity of extensive lymphadenectomy. The aim of this study was to analyze the prognostic factors and to develop a prognostic model of extraregional lymphadenectomy for colon cancer with isolated PALNM.

\section{Materials and Methods}

\section{Patients}

From our prospectively collected database, a total of 1,082 patients who underwent curative surgery for colonic adenocarcinoma with pathological lymph node metastasis at Severance Hospital, Yonsei University, Seoul, South Korea, were included. With the following exclusion criteria, 49 patients who received a pathologic diagnosis of isolated synchronous PALNM were included in this study: (1) distant metastases other than to PALNM, (2) lateral pelvic lymph node metastasis, and (3) nodal metastasis above the renal vessels. Clinicopathologic data including age, sex, body mass index, location of tumor, preoperative carcinoembryonic antigen (CEA), postoperative chemotherapy, pathologic tumor and nodal stage, tumor differentiation, the numbers of total and positive PALN, and survival data were obtained.

\section{Preoperative staging and treatment}

Preoperative staging evaluation was performed using chest X-ray and abdominal and chest computed tomography (CT) scan. For PALNM diagnosis, abdominal CT scan was mainly used and positron emission tomogram (PET) was performed in selected cases where the diagnosis of PALNM by CT was uncertain. We considered a PALN to be radiologically positive if a lymph node at the region between the left renal vein and the bilateral common iliac vessels was $0.5 \mathrm{~cm}$ at the short-axis diameter, with spiculated borders or showing a mottled heterogenic pattern according to the preoper- ative abdominal CT scan or had fludeoxyglucose uptake on PET-CT. In addition to the standard extent of lymph node dissection, all lymphovascular tissues along the aortocaval area between the left renal vein and the bilateral common iliac vessels were removed in patients with advanced colon cancer with radiologically suspected metastatic PALN. Postoperative chemotherapy (5-fluorouracil/leucovorin regimen or oxaliplatin, folinic acid and 5-fluorouracil [FOLFOX]) was administered 3-4 weeks after surgery for 6 months.

\section{Evaluation parameters and statistical analyses}

The classification system of the AJCC sixth edition was used to determine pathological tumor depth ( $\mathrm{pT}$ ), the number of metastasized lymph nodes ( $\mathrm{pN})$, and cancer stage. PALN was defined as the presence of involved lymph nodes in the region between the bilateral common iliac vessels and

Table 1. Characteristics of colon cancer patients with para-aortic lymph node metastasis

\begin{tabular}{|c|c|}
\hline Characteristic & No. $(\%)(n=49)$ \\
\hline \multicolumn{2}{|l|}{ Sex } \\
\hline Male & $29(59.2)$ \\
\hline Female & $20(40.8)$ \\
\hline Age, mean $\pm S D(y r)$ & $57.5 \pm 11.5$ \\
\hline Body mass index, mean $\pm S D\left(\mathrm{~kg} / \mathrm{m}^{2}\right)$ & $23.7 \pm 2.8$ \\
\hline \multicolumn{2}{|l|}{ Location of tumor } \\
\hline Cecum & $1(2.0)$ \\
\hline Ascending colon & $5(10.2)$ \\
\hline Descending colon & $5(10.2)$ \\
\hline Sigmoid colon & $38(77.6)$ \\
\hline Preoperative CEA, mean \pm SD $(\mathrm{ng} / \mathrm{mL})$ & $23.7 \pm 37.4$ \\
\hline POD 7 CEA, mean \pm SD $(\mathrm{ng} / \mathrm{mL})$ & $7.4 \pm 13.0$ \\
\hline Adjuvant chemotherapy & $47(95.9)$ \\
\hline \multicolumn{2}{|l|}{ Tumor stage } \\
\hline $\mathrm{T} 2$ & $1(2.0)$ \\
\hline T3 & $43(87.8)$ \\
\hline $\mathrm{T} 4$ & $5(10.2)$ \\
\hline \multicolumn{2}{|l|}{ Histology } \\
\hline Well differentiated & $4(8.2)$ \\
\hline Moderately differentiated & $34(69.4)$ \\
\hline Poorly differentiated & $6(12.2)$ \\
\hline Mucinous & $5(10.2)$ \\
\hline Total retrieved LNs, mean $\pm \mathrm{SD}$ & $38.2 \pm 22.0$ \\
\hline Positive retrieved LNs, mean \pm SD & $15.6 \pm 15.1$ \\
\hline Total para-aortic LNs, mean \pm SD & $6.9 \pm 5.2$ \\
\hline Positive para-aortic LNs, mean \pm SD & $3.9 \pm 4.0$ \\
\hline
\end{tabular}

SD, standard deviation; CEA, carcinoembryonic antigen; POD, postoperative day; LN, lymph node. 
left renal vein. All patients received postoperative clinical examinations, measurement of serum CEA levels, and chest X-rays every 3 months, and a chest/abdominal CT every 6 months over a period of 3 years. After 3 years, the follow-up interval was changed to 6 months. Recurrence was defined as the presence of a histologically and / or radiologically confirmed tumor. Overall survival (OS) time was calculated from the date of surgery to the date of the latest follow-up visit or the date of death due to any cause, and disease-free survival (DFS) time was defined as the time from surgery to any type of recurrence. Patients who died from other causes or were alive without progression or recurrence at the most recent follow-up were treated as censored in the analysis of DFS time. Five-year survival rate was determined by the Kaplan-Meier method, and the log-rank test was used to compare survival rates among subgroups. The log-rank test was used for univariate analysis and independent prognostic factors were identified by multivariate analysis using the Cox proportional hazards model to calculate hazard ratios (HR). Age ( $\leq 60$ or $>60$ years), sex (female or male), tumor depth (T1, T2 or T3, T4), number of positive PALNs $(\leq 7$ or $>7)$, and preoperative CEA level $(\leq 5$ or $>5)$ were included as covariates. The results of the Cox model analysis are reported using $\mathrm{HR}$ and $95 \%$ confidence intervals (CI). All statistical tests were performed using IBM SPSS ver. 21.0 (IBM Corp., Armonk, NY). Data are expressed as means with standard deviation. $\mathrm{p}$-values of less than 0.05 were defined to indicate a statistically significant difference.

\section{Ethical statement}

The study was approved by the Institutional Review Board of Severance Hospital (IRB No. 2017-2432-001) and performed in accordance with the principles of the Declaration of Helsinki. The informed consent was waived.

\section{Results}

\section{Patient characteristics}

Table 1 summarizes the characteristics of the 49 patients with pathologically positive PALN who underwent PALND for colonic cancer. The mean age was $57.5 \pm 11.5$ years; 20 patients $(40.8 \%)$ were women and $29(59.2 \%)$ men. Body mass index was $23.7 \pm 2.8$. One tumor $(2.0 \%)$ was located in the cecum, five $(10.2 \%)$ in the ascending colon, five $(10.2 \%)$ in the descending colon, and $38(77.6 \%)$ in the sigmoid colon. The mean CEA level before the operation was $23.7 \pm 37.4$ $\mathrm{ng} / \mathrm{mL}$ and the CEA level measured 7 days after curative resection was $7.4 \pm 13.0 \mathrm{ng} / \mathrm{mL}$. Of the total 49 patients, $47(95.9 \%)$ received chemotherapy after curative resection.

Table 2. Prognostic factors of 5-year survival by univariate analysis

\begin{tabular}{|c|c|c|c|c|c|}
\hline Prognostic factor & No. $(n=49)$ & OS $(\%)$ & p-value & DFS $(\%)$ & p-value \\
\hline \multicolumn{6}{|l|}{ Age (yr) } \\
\hline$\leq 60$ & 28 & 30.8 & 0.813 & 19.8 & 0.830 \\
\hline$>60$ & 21 & 31.4 & & 28.6 & \\
\hline \multicolumn{6}{|l|}{ Sex } \\
\hline Male & 29 & 28.5 & 0.257 & 22.2 & 0.872 \\
\hline Female & 20 & 35.0 & & 25.0 & \\
\hline \multicolumn{6}{|l|}{ Tumor stage } \\
\hline $\mathrm{T} 1$ and $\mathrm{T} 2$ & 6 & 33.3 & 0.954 & 33.3 & 0.584 \\
\hline $\mathrm{T} 3$ and $\mathrm{T} 4$ & 43 & 30.7 & & 21.7 & \\
\hline \multicolumn{6}{|l|}{ Histology } \\
\hline Well and moderately & 43 & 31.7 & 0.590 & 22.5 & 0.667 \\
\hline Poorly and mucinous & 6 & 25.0 & & 33.3 & \\
\hline \multicolumn{6}{|l|}{ Positive retrieved PALNs } \\
\hline$\leq 7$ & 42 & 36.5 & 0.010 & 27.5 & 0.027 \\
\hline$>7$ & 7 & 14.3 & & 14.3 & \\
\hline \multicolumn{6}{|l|}{ Preoperative CEA } \\
\hline$\leq 5$ & 19 & 46.3 & 0.122 & 41.4 & 0.039 \\
\hline$>5$ & 30 & 21.5 & & 11.7 & \\
\hline
\end{tabular}

OS, overall survival; DFS, disease-free survival; PALN, para-aortic lymph node; CEA, carcinoembryonic antigen. 
A
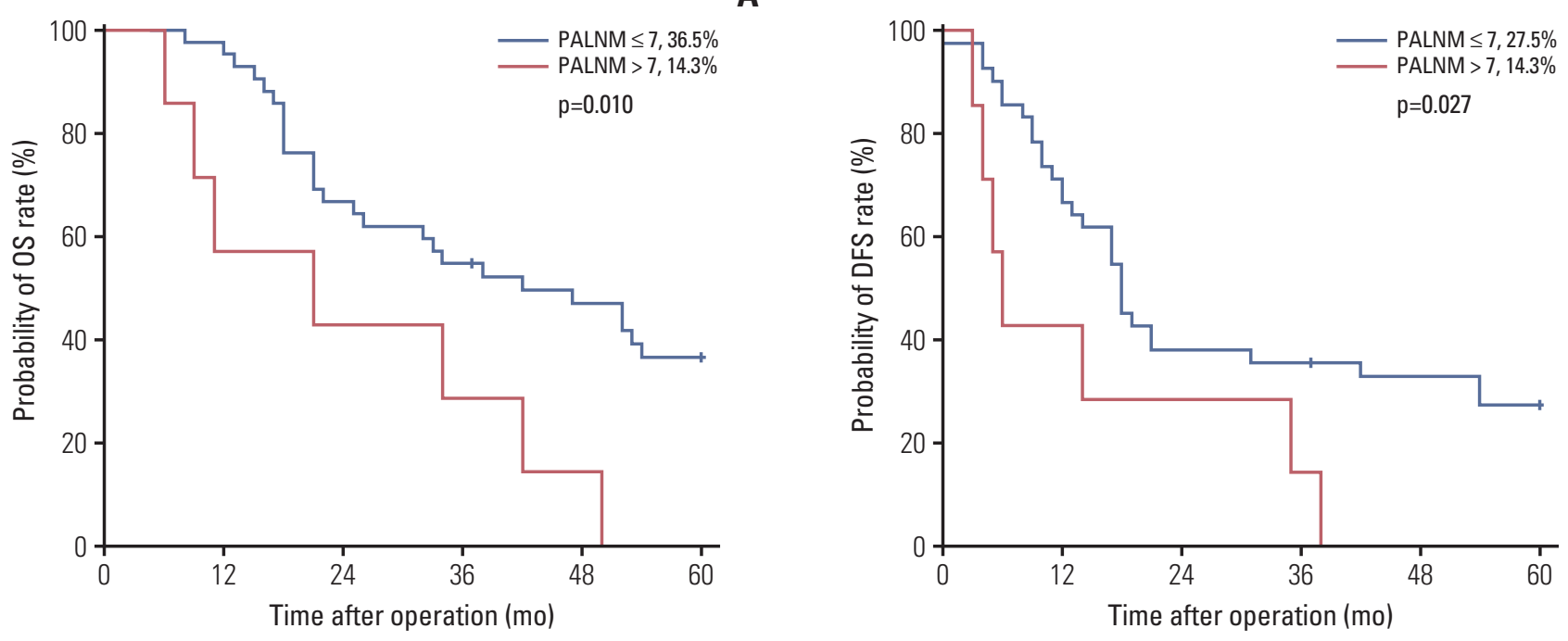

C
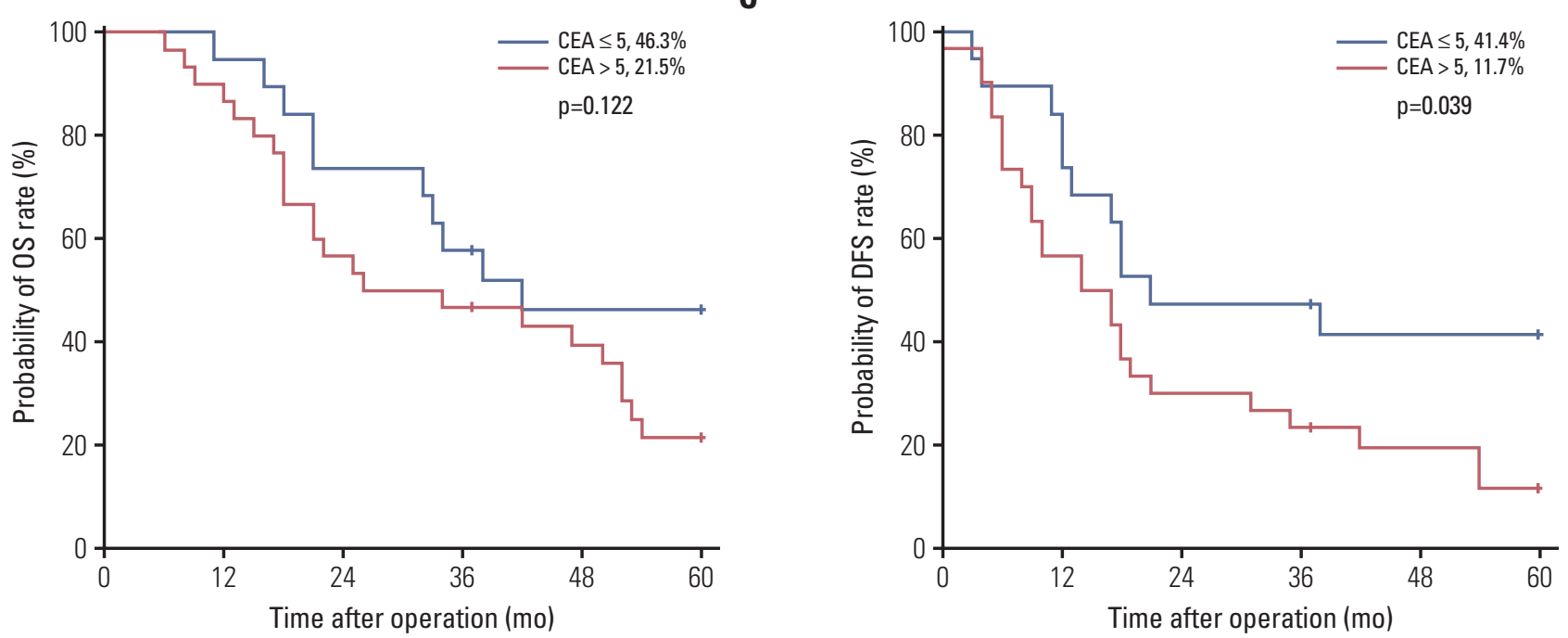

Fig. 1. (A) Five-year overall survival (OS) of patients with para-aortic lymph node metastasis (PALNM) according to the number of positive para-aortic lymph node (PALN). (B) Five-year disease-free survival (DFS) according to the number of positive PALN. (C) Five-year OS according to the preoperative carcinoembryonic antigen (CEA) level. (D) Five-year DFS according to the preoperative CEA level. (Continued to the next page)

\section{Pathologic outcomes}

Pathologic characteristics of the tumors are summarized in Table 1. There was one T2 tumor $(2.0 \%), 43 \mathrm{~T} 3$ tumors $(87.8 \%)$, and five $\mathrm{T} 4$ tumors $(10.1 \%)$. The moderately differentiated type $(69.4 \%)$ was the most common histological type, followed by poorly differentiated $(12.2 \%)$, mucinous
(10.2\%), and well differentiated (8.2\%). The mean numbers of total and positive lymph nodes were $38.2 \pm 22.0$ and $15.6 \pm 15.1$, respectively. The mean numbers of total harvested and metastatic PALNs were $6.9 \pm 5.2$ and $3.9 \pm 4.0$, respectively. 
$\mathbf{E}$
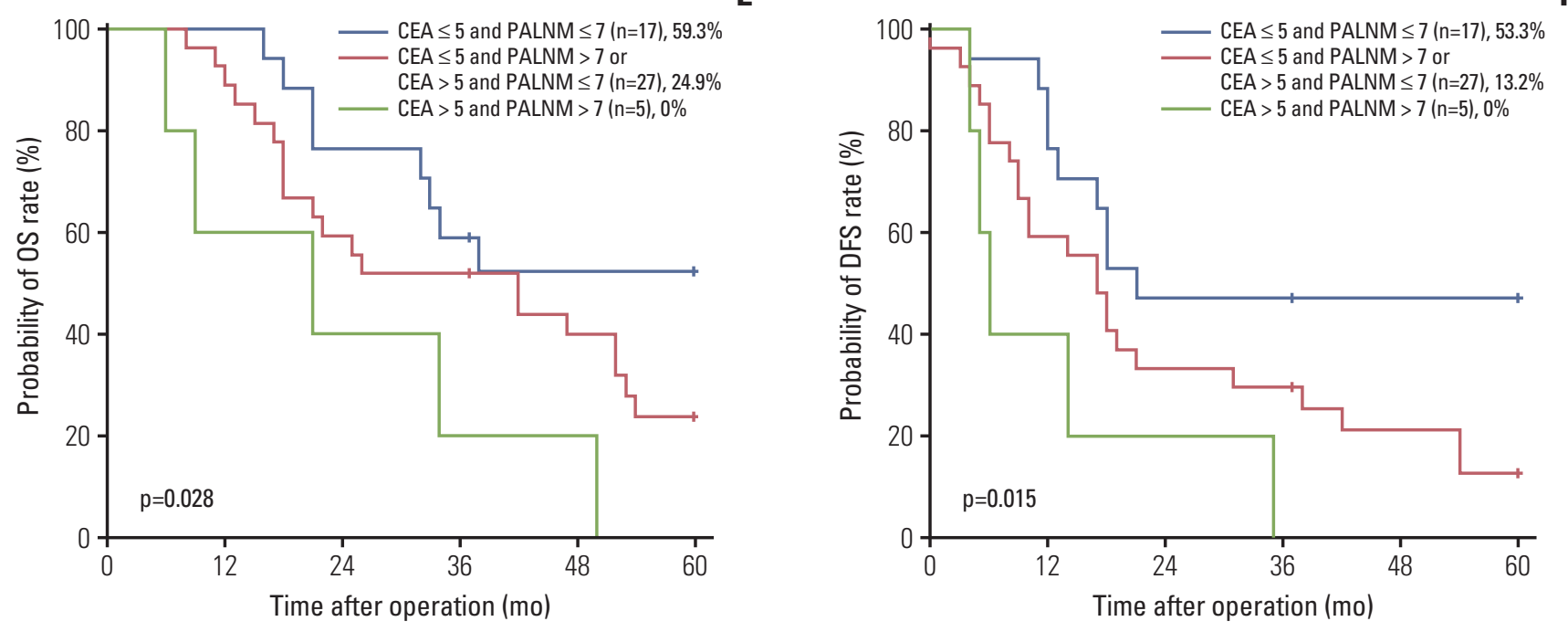

Fig. 1. (Continued from the previous page) (E) Five-year OS according to the prognostic model. (F) Five-year DFS according to the prognostic model.

Table 3. Prognostic factors of 5-year survival by multivariate analysis

\begin{tabular}{|c|c|c|c|c|}
\hline \multirow{2}{*}{ Prognostic factor } & \multicolumn{2}{|c|}{ Overall survival } & \multicolumn{2}{|c|}{ Disease-free survival } \\
\hline & HR $(95 \%$ CI $)$ & p-value & HR $(95 \%$ CI) & p-value \\
\hline \multicolumn{5}{|l|}{ Age } \\
\hline$>60 \mathrm{yr}$ vs. $\leq 60 \mathrm{yr}$ & $0.885(0.434-1.806)$ & 0.738 & $1.049(0.534-2.061)$ & 0.889 \\
\hline \multicolumn{5}{|l|}{ Sex } \\
\hline Female vs. male & $0.745(0.353-1.571)$ & 0.440 & $0.859(0.416-1.773)$ & 0.682 \\
\hline \multicolumn{5}{|l|}{ Tumor stage } \\
\hline $\mathrm{T} 3$ and $\mathrm{T} 4 \mathrm{vs} . \mathrm{T} 1$ and $\mathrm{T} 2$ & $0.816(0.276-2.413)$ & 0.713 & $1.108(0.382-3.217)$ & 0.850 \\
\hline \multicolumn{5}{|l|}{ Histology } \\
\hline Poorly and mucinous vs. well and moderately & $1.660(0.553-4.986)$ & 0.366 & $1.299(0.443-3.807)$ & 0.634 \\
\hline \multicolumn{5}{|l|}{ Positive retrieved PALNs } \\
\hline$>7$ vs. $\leq 7$ & $3.291(1.309-8.275)$ & 0.011 & $2.484(0.993-6.211)$ & 0.052 \\
\hline \multicolumn{5}{|l|}{ Preoperative CEA } \\
\hline$>5$ vs. $\leq 5$ & $1.575(0.738-3.361)$ & 0.241 & $1.953(0.940-4.057)$ & 0.073 \\
\hline
\end{tabular}

HR, hazard ratio; CI, confidence interval; PALN, para-aortic lymph node; CEA, carcinoembryonic antigen.

\section{Univariate and multivariate survival analyses of prog- nostic factors}

The median survival time was 37 months (range, 6 to 169 months). Five-year OS and DFS rates were $33.9 \%$ and $26.5 \%$, respectively. Table 2 summarizes the univariate analysis. Univariate analyses revealed that the number of positive PALN and the preoperative CEA level were significantly associated with OS and DFS (Table 2). Fig. 1 describes patient survival according to the number of positive PALN and preoperative CEA level. The 5-year OS and DFS rates were higher in patients with $\leq 7$ positive PALN $(36.5 \%$ and $27.5 \%)$ than in those with $>7$ PALN (14.3\% and 14.3\%; $\mathrm{p}=0.010$ and $\mathrm{p}=0.027$, respectively) (Fig. 1A and B). Preoperative CEA level $>5$ also adversely affected patient prognosis, with a lower 5-year OS and DFS rate at $21.5 \%$ and $11.7 \%$ observed in patients with CEA level $>5$ compared to those with CEA level $\leq 5$ (collectively at $46.3 \%$ and $41.4 \%, p=0.122$ and 
$\mathrm{p}=0.039$ ) (Fig. 1C and D). Multivariate analysis showed the number of positive PALN (HR, 3.291; 95\% CI, 1.309 to 8.275; $\mathrm{p}=0.011$ ) was an independent prognostic factor for $\mathrm{OS}$ and the number of positive PALN (HR, 2.484; 95\% CI, 0.993 to 6.211; $\mathrm{p}=0.052$ ) and preoperative CEA level (HR, 1.953; 95\% CI, 0.940 to 4.057 ; $\mathrm{p}=0.073$ ) as marginally independent prognostic factors for DFS (Table 3). According to our prognostic model with two prognostic factors, the 5-year OS and DFS rate increased to $59.3 \%$ and $53.3 \%$, respectively, in patients with $\leq 7$ positive PALN and a CEA level $\leq 5$ (Fig. $1 \mathrm{E}$ and $\mathrm{F}$ ). The 5-year OS and DFS rates of patients with CEA level $\leq 5$ and $>7$ positive PALN or CEA level $>5$ and $\leq 7$ positive PALN were $24.9 \%$ and $13.2 \%$, respectively. The 5-year OS and DFS rates of patients with CEA level $>5$ and $>7$ positive PALN were $0 \%$ and $0 \%$, respectively.

\section{Discussion}

In the context of hepatic metastasis in colorectal cancer, upfront primary tumor resection with metastasectomy is recommended in surgically amenable metastasis (lesions $\leq 4$, absence of bilobar metastasis, and radiologically negative periportal lymph nodes) [5]. In the current prognostic analyses of 49 colon cancer patients with pathological PALNM who underwent extraregional lymphadenectomy, our results show that PALND may be justified in highly selected patients. We have found that an elevated preoperative CEA level and a greater number of positive PALN indicates a poor survival outcome and the subgroup of patients with $\leq 7$ positive PALN and a CEA level $\leq 5$ might still benefit from surgery. Thus, systematic PALND should be considered as a treatment option in those patients.

Ogura et al. [6] attempted to establish a prognostic model for visible PALNM with CRC and reported that lymphovascular invasion, nodal status, elevated preoperative CEA level, and visible PALNs $\geq 10 \mathrm{~mm}$ were independent prognostic factors for patients with visible PALNs, although that model could not predict pathologic PALNM. Choi et al. [1] observed that the presence of two or fewer PALN metastases was a good indication for PALND in 24 CRC patients with isolated PALNM based on their univariate analysis; however, there were no data regarding pathologic PALNM and no significant prognostic factors were identified by multivariate analysis. In our study, we tried to develop a prognostic model using multivariate analysis for pathologically identified PALNM after extensive lymphadenectomy in colon cancer.

Preoperative serum levels of the tumor marker CEA, which represent serum tumor burden, are of prognostic significance. CEA levels $\geq 5.0 \mathrm{ng} / \mathrm{mL}$ have an adverse effect on survival that is independent of tumor stage [7-9]. Hokuto et al. [10] reported that a serum CEA level of $\geq 20 \mathrm{ng} / \mathrm{mL}$ was one of the prognostic factors for DFS in patients with $\geq 4$ colorectal liver metastases and a systematic review assessing prognostic factors for patients undergoing lung metastasec-

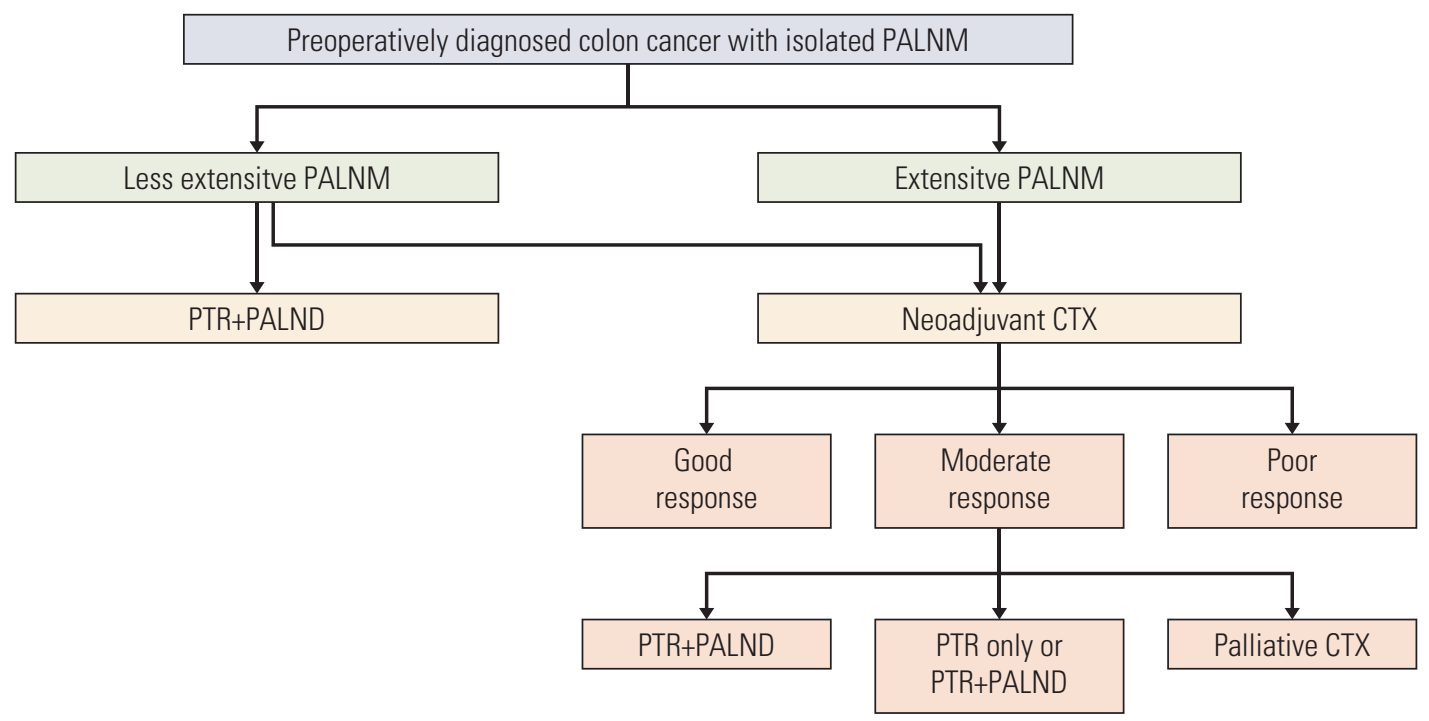

Fig. 2. Suggested treatment strategy for colon cancer with isolated para-aortic lymph node metastasis. PALNM, para-aortic lymph node metastasis; PTR, primary tumor resection; PALND, para-aortic lymph node dissection; CTX, chemotherapy. 
tomy showed that a prethoracotomy associated elevation in CEA level was associated with poor survival [11]. In this study, elevated preoperative CEA level was identified to be an independent risk predictor for oncologic outcomes, which is consistent with the prior results in the literature.

Lymph node involvement is one of the strongest predictors of outcome following surgical resection of CRC [12-16]. Moreover, we found that the long-term oncologic outcomes were significantly better in the regional lymph node metastasis group than in the PALNM group, which means that the location of the metastatic lymph node also matters [4]. In the present study, the 5-year survival rate was poor in patients with a large number of positive PALN, which was identified as an independent prognostic factor after PALND. Thus, PALND should be considered as a treatment option in patients with a relatively smaller number of infiltrated lymph nodes.

The availability of increasingly effective systemic chemotherapy has prompted interest in preoperative or neoadjuvant chemotherapy for stage IV CRC [17]. Neoadjuvant chemotherapy might play a role in evaluating the natural history of metastatic disease, which then enables the surgeon to decide whether to proceed with surgery or to continue palliative chemotherapy in cases of CRC with PALNM. An expert review further emphasized the role of neoadjuvant chemotherapy in the selection of candidates for PALND because tumor regression after treatment may imply a favorable biology, thereby selecting for patients more likely to benefit from PALND [18]. Currently, we have discussed treatment plans based on a multidisciplinary approach for these patients and most of them were recommended to receive upfront systemic chemotherapy followed by surgical resection for both the primary tumor and extraregional lymph nodes (PALN).

A multimodality approach including perioperative chemotherapy may be needed to improve survival of patients with colon cancer with isolated PALNM and the treatment strat- egy should be individualized according to the characteristics of the disease. Our results suggest that upfront primary tumor resection with extended lymphadenectomy may be justified in patients with low CEA levels and less extensive PALNM, based on our prognostic model (Fig. 2). Neoadjuvant chemotherapy can be an initial targeted treatment for colon cancer with an elevated CEA level or extensive PALNM. Primary tumor resection with or without PALND should be performed after neoadjuvant treatment based on tumor response. Treatment of progressive disease with elevated CEA level and extensive PALNM even after neoadjuvant chemotherapy needs to be converted to palliative chemotherapy with other additional treatments such as intensity-modulated radiotherapy, tomotherapy, and robotic linear accelerators.

There are several limitations to this study, including a single institutional experience, the small sample size due to the rarity of this metastatic pattern, and its retrospective analysis. In addition, new biologic chemotherapeutic regimens were not used in most of the patients, and therefore, we could not analyze the prognostic factors after neoadjuvant treatment.

PALND might be beneficial in carefully selected patients with low CEA levels and less extensive PALNM.

\section{Conflicts of Interest}

Conflict of interest relevant to this article was not reported.

\section{Acknowledgments}

This work was supported by the National Research Foundation of Korea (NRF) Grant funded by the Korea Government (MSIP) (No. 2014R1A5A2010008).

This work was supported by the Student's Association of the Graduate School of Yonsei University funded by the Graduate School of Yonsei University.

\section{References}

1. Choi PW, Kim HC, Kim AY, Jung SH, Yu CS, Kim JC. Extensive lymphadenectomy in colorectal cancer with isolated paraaortic lymph node metastasis below the level of renal vessels. J Surg Oncol. 2010;101:66-71.

2. Gagniere J, Dupre A, Chabaud S, Peyrat P, Meeus P, Rivoire M. Retroperitoneal nodal metastases from colorectal cancer: curable metastases with radical retroperitoneal lymphadenectomy in selected patients. Eur J Surg Oncol. 2015;41:731-7.

3. Greene FL. The American Joint Committee on Cancer: updat- ing the strategies in cancer staging. Bull Am Coll Surg. 2002;87:13-5.

4. Bae SU, Han YD, Cho MS, Hur H, Min BS, Baik SH, et al. Oncologic outcomes of colon cancer patients with extraregional lymph node metastasis: comparison of isolated paraaortic lymph node metastasis with resectable liver metastasis. Ann Surg Oncol. 2016;23:1562-8.

5. Khatri VP, Petrelli NJ, Belghiti J. Extending the frontiers of surgical therapy for hepatic colorectal metastases: is there a limit? 
J Clin Oncol. 2005;23:8490-9.

6. Ogura A, Akiyoshi T, Takatsu Y, Nagata J, Nagasaki T, Konishi $\mathrm{T}$, et al. The significance of extended lymphadenectomy for colorectal cancer with isolated synchronous extraregional lymph node metastasis. Asian J Surg. 2017;40:254-61.

7. Wiggers T, Arends JW, Volovics A. Regression analysis of prognostic factors in colorectal cancer after curative resections. Dis Colon Rectum. 1988;31:33-41.

8. Park IJ, Choi GS, Lim KH, Kang BM, Jun SH. Serum carcinoembryonic antigen monitoring after curative resection for colorectal cancer: clinical significance of the preoperative level. Ann Surg Oncol. 2009;16:3087-93.

9. Thirunavukarasu P, Sukumar S, Sathaiah M, Mahan M, Pragatheeshwar KD, Pingpank JF, et al. C-stage in colon cancer: implications of carcinoembryonic antigen biomarker in staging, prognosis, and management. J Natl Cancer Inst. 2011;103: 689-97.

10. Hokuto D, Nomi T, Yamato I, Yasuda S, Obara S, Yoshikawa $\mathrm{T}$, et al. The prognosis of liver resection for patients with four or more colorectal liver metastases has not improved in the era of modern chemotherapy. J Surg Oncol. 2016;114:959-65.

11. Gonzalez M, Poncet A, Combescure C, Robert J, Ris HB, Gervaz P. Risk factors for survival after lung metastasectomy in colorectal cancer patients: a systematic review and metaanalysis. Ann Surg Oncol. 2013;20:572-9.

12. Tepper JE, O'Connell MJ, Niedzwiecki D, Hollis D, Compton $\mathrm{C}$, Benson AB 3rd, et al. Impact of number of nodes retrieved on outcome in patients with rectal cancer. J Clin Oncol. 2001;19:157-63.

13. Chang GJ, Rodriguez-Bigas MA, Skibber JM, Moyer VA. Lymph node evaluation and survival after curative resection of colon cancer: systematic review. J Natl Cancer Inst. 2007;99: 433-41.

14. Le Voyer TE, Sigurdson ER, Hanlon AL, Mayer RJ, Macdonald JS, Catalano PJ, et al. Colon cancer survival is associated with increasing number of lymph nodes analyzed: a secondary survey of intergroup trial INT-0089. J Clin Oncol. 2003;21:2912-9.

15. Jang KU, Kim CW, Kim KH, Lim SB, Yu CS, Kim TW, et al. Prognostic factors in terms of the number of metastatic nodules in patients with colorectal cancer liver metastases. Ann Coloproctol. 2016;32:92-100.

16. Park SJ, Lee KY, Kim SY. Clinical significance of lymph node micrometastasis in stage I and II colon cancer. Cancer Res Treat. 2008; 40:75-80.

17. Nordlinger B, Sorbye H, Glimelius B, Poston GJ, Schlag PM, Rougier $\mathrm{P}$, et al. Perioperative FOLFOX4 chemotherapy and surgery versus surgery alone for resectable liver metastases from colorectal cancer (EORTC 40983): long-term results of a randomised, controlled, phase 3 trial. Lancet Oncol. 2013;14: 1208-15.

18. Albandar MH, Cho MS, Bae SU, Kim NK. Surgical management of extra-regional lymph node metastasis in colorectal cancer. Expert Rev Anticancer Ther. 2016;16:503-13. 